\title{
Probiotics as Therapeutics
}

\author{
Shiwangi Morya, Gauri Aeron* \\ Department of Biotechnology, SD College of Engineering \&Technology, Muzaffarnagar-25100, India
}

Received: 9 November, 2017; Accepted: 30 November, 2017; Published: 12 December, 2017

*Corresponding author: Gauri Aeron, Department of Biotechnology, S.D. College of Engineering \&Technology, Muzaffarnagar-25100, India, Tel: +919557190990; E-mail: gauriaeron@gmail.com

\begin{abstract}
There is an increasing scientific and commercial interest in the use of valuable microorganisms, or "probiotics," for the averting and treatment of disease. The microorganisms most frequently used as probiotic agents are lactic-acid bacteria such as Lactobacilli species, and many other species are also used such as Bifidobacteria species, saccharomyces cerevisie var. bouladi and many more which has been extensively studied as probiotic species. Multiple mechanisms of action have been hypothesized, including lactose digestion, production of antimicrobial agents (such as bacteriocin protein compound), competition for space or nutrients, and immunomodulation. We have reviewed recent studies of probiotics for the treatment and manage of infectious diseases. Studies of pediatrics diarrhea show considerable evidence of clinical benefits from probiotic therapy in patients with viral gastroenteritis, and data treatment for Clostridium difficile diarrhea appear promising. However, data to support use of probiotics for prevention of traveler's diarrhea are more limited. New research suggests eventual applications in vaccine expansion and prevention of sexually transmitted diseases. Further studies are needed to take full advantage of this conventional medical approach and to apply it to the infectious diseases of the new millennium.
\end{abstract}

\section{Introduction}

Probiotics are living microorganisms that help us to stay healthy which when ingested have valuable effects on the equilibrium and the physiological functions of the human intestinal microflora by various ways. Probiotics have been recently defined as "live microbes which transit the gastrointestinal tract and in doing so profit the health of the consumer differing from the earlier definitions which focused on probiotic interactions with aboriginal intestinal microbes. These definitions of probiotic bacteria generally agree that probiotic bacteria should be living organisms to furnish health benefits. Probiotics have been reported to play a therapeutic role by enhancing immunity, lowering cholesterol, improving lactose tolerance and preventing some types of cancers such as colon cancer and many more. In the recent past, there has been an surge of probiotic-based health products mostly in the form of fermented dairy products as well as nutritional supplements. The markets for probiotic products and supplements are growing worldwide because of various benefits. Today there are more than 80 "Bifidus"and "Acidophilus"-containing products worldwide, including a number of fermented dairy products. Capability of probiotic bacteria (such as lactobacilli, bifidibateria and many other species of different bacteria) in a product at the point of operation is an most important consideration for their effectiveness, as they have to survive during the processing and shelf life of food and supplements, transit through high acid conditions of the stomach and enzymes and bile salts in the small intestine very efficiently. The consumption of probiotics at a level of $10^{8}-10^{9} \mathrm{cfu} / \mathrm{g}$ per day is a generally quoted figure for ample probiotic consumption, equating to $100 \mathrm{~g}$ of a food product with $10^{6}-10^{7} \mathrm{cfu} / \mathrm{g}$. Analysis of probiotic products in many different countries has established that probiotic strains exhibit poor survival in conventional fermented dairy products. The probiotic preparations such as tablets, powders etc. may contain lesser viable counts. Of the 15 feed supplements examined, viable probiotic counts varied to a great extent with 3 products containing no lactobacillus species at all, although the supplements were supposed to contain $\mathrm{L}$. acidophilus. Probiotic survival in products is affected by a range of factors including $\mathrm{pH}$, acidification (during storage) in fermented products, hydrogen peroxide production, oxygen toxicity (oxygen permeation through packaging), storage temperatures, stability in dried or frozen form, poor growth in milk, lack of proteases to break down milk protein to simpler nitrogenous substances and compatibility with traditional starter culture during fermentation (Dave and Shah, 1997a, b, c; Kailasapathy and Rybka, 1997; Shah, 2000). Oxygen plays a vital role in the poor survival of probiotic bacteria. Encapsulation of probiotic bacteria is an alternative that provides protection for living cells bare to an adverse environment. It also helps food materials to oppose processing and packaging conditions, improving flavou, aroma, stability, nutritional value and product appearance.

\section{How antibiotics imbalance gut microbiota}

The gastrointestinal tract is one of the chief interfaces between the human internal atmosphere and the outside world. Its function is to digest and take up the vital nutrients offer by food. At the equivalent time, it also provides a barrier that prevent health intimidating molecules to pass through the intestinal mucosa and access the systemic circulation. Intestinal dysfunctions are now supposed to be contributing factors to many chronic diseases such as allergies, autoimmune disorders, inflammatory disorders and degenerative diseases. 


\section{Intestinal dysbiosis}

Intestinal microflora signify an ecosystem of the highest intricacy. The microflora not only has a decisive role in the digestion and absorption of nutrients, in the amalgamation of vitamins and fatty acids, in the detoxification of ingested chemicals but also in the command of the immune system. Alterations in the composition of the microflora may therefore have severe penalty for the hosts' health. A frequent disorder of the intestinal function is dysbiosis. This is an overgrowth/ overproduction of pathogenic bacteria in the intestine. The adult human intestinal tract is estimated to host up 55 different genera of bacteria, accounting for more than 501 different species.

- Antibiotic use is a common cause of major modification Dosage, length of administration, spectrum of activity will conclude the impact on the microbial flora.

- Psychological stress can also affect the composition of the flora, including a noteworthy diminish in beneficial bacteria (Lactobacilli and Bifidobacteria) and an increase in pathogenic $E$. coli. Stress may affect bacterial growth by significantly tumbling the mucosal production of mucopolysaccharides and mucins, which are important for hinder the adherence of pathogenic organisms, and by decreasing the production of immunoglobulin A $(\operatorname{IgA})$, which play a crucial role in their elimination. Neurochemicals twisted upon psychological stress can also nonstop enhance the growth of pathogenic organisms: norepinephrine stimulates the growth of Y. Enterocolitica, P. Aeruginosa, and gram-negative bacteria such as E. coli.

- Another factor that may have an impact on the human intestinal flora is diet. Some diets encourage the enlargement of beneficial microorganisms, while others promote harmful microfloral activities. For instance, diets rich in sulfur compounds (dairy products, eggs, certain vegetables, dried fruits...) promote the growth of sulfate-reducing bacteria. Globally it emerge that populations consuming the typical Western diet have more anaerobic bacteria, less Enterococci, and less types of yeasts than populations uncontrollable a vegetarian or high complexcarbohydrate diet.

\section{Intestinal permeability: leaky gut syndrome}

The healthy intestinal mucosa normally take up small molecules that result from complete digestion. Intestinal cells articulate specialized carrier protein that bring nutrients through the intestinal wall and into the bloodstream. Bigger molecules will not be transported by these systems and are normally kept within the gut for the reason that the intestine mucosal cells are tightly packed together. Leaky gut syndrome (LGS) is a condition in which the ability of the intestinal wall to keep out huge and unwanted molecules, is reduced. When the places between the cells of the intestinal wall become enlarged, macromolecules, antigens and toxins will make their way into the bloodstream.

\section{What causes Leaky Gut Syndrome}

\section{A large numeral of factors can lead to leaky gut}

- Dietary components: Fermentation of certain dietary components (proteins, refined carbohydrates) leads to potentially injurious end-products: ammonia, amines, phenols, sulfides... these compounds diminish the life-span of mucosal cells. Food additives, alcohol, caffeine also annoy the gut wall.

- Gut dysbiosis: Production of toxic compounds through fermentation also depends on the type of bacteria present in the bowel. In case of dysbiosis, overgrown pathogenic bacteria create toxins and compounds that are very detrimental to intestinal cells. For instance, sulfate-reducing bacteria produce toxic hydrogen sulfide.

- Food allergies and intolerances: Intolerance to certain food (lactose...) can lead to destructive gut inflammation.

- Chronic stress: In addition to favoring dysbiosis, stress diminish blood flow to the gut leaving it unable to repair itself. Stress also causes the cells of the intestine to contract which results in larger gaps stuck between cells.

\section{Consequences of Leaky Gut}

Altered permeability of the intestinal wall can have highly harmful effects, including

- Nutritional deficiencies: The carrier systems that usually transfer the nutrients through the intestinal wall are less active in damaged or inflamed mucosal cells.

- Increased absorption of environmental toxins: The gut mucosa is usually an efficient barrier against environmental chemicals that are present in food (food additives, pesticides, PCBs...). When allowed to pass into the circulation, these toxins can cause harm to all organs, notably the liver and the brain. Multiple Chemical Sensitivities may develop as the nervous system becomes sensitized.

- Development of allergies and auto-immune reactions: Undigested, large molecules pass into the bloodstream. The immune system identify these molecules as foreign and raises immunoglobulins against them. As a result, affected patients will develop allergies to many types of foods, which actually initiates a vicious cycle, since allergies will cause gut inflammation that conduct to more intestinal permeability...In addition, some of the molecules that pass into the blood may share homologies with proteins that are normally present in the body. Antibodies against these molecules will therefore attack the body's own cells, leading to auto-immune diseases.

- Chronic activation (inflammation) of the immune system: One bacterial compound that can easily make its way to the blood is lipopolysaccharide (LPS). Present in the bloodstream LPS will encourage a strong pro-inflammatory response in monocytes and macrophages, involving recognition by a receptor (Toll-like receptor-4) and the subsequent secretion of cytokines such as IL-1, IL-6, TNF-alpha. Such chronic inflammatory condition is observed Chronic Fatigue Syndrome (CFS/ME). LPS also induces the NK-kB-mediated production of nitric oxide. Because NO is amplified, NK function is inhibited and opportunistic infections such as mycoplasma infections are often observed. Herpesviruses, which tend to reactivate in a context of immune activation, will also be frequently detected. 


\section{How do probiotics work?}

The screening process of probiotics which has been explained by the following mechanism:

- Exertion for nutrients - Within the gut valuable and pathogenic micro-organisms will be utilising the equivalent types of nutrients. This results in a general competition between various types of bacteria for these nutrients. When a probiotic is taken there is an overall decline in nutrients available/ competition of nutrients for pathogenic bacteria and as a result this minimises or decreases the levels of pathogenic/ infectious micro-organisms effectively.

- Competition for adhesion sites - Beneficial bacteria can affix to the gut wall and form colonies at various places throughout the gut. This ruin pathogenic/ infectious bacterium from gaining a foothold, ensuing in their eviction from the body.

- Augmentation in digestion - Probiotics have been exposed to increase the efficacy of digestion and therefore provide an step up in digestion.

- Lactic acid fabrication - Probiotics produce lactic acid which take action to reduce the gut $\mathrm{pH}$, inhibiting the growth of pathogenic bacteria, which prefer a additional alkaline environment.

- Effect on immunity - Probiotics have been shown to boost the levels of cell-signalling chemicals and the efficacy of infectionfighting cells (white blood cells)

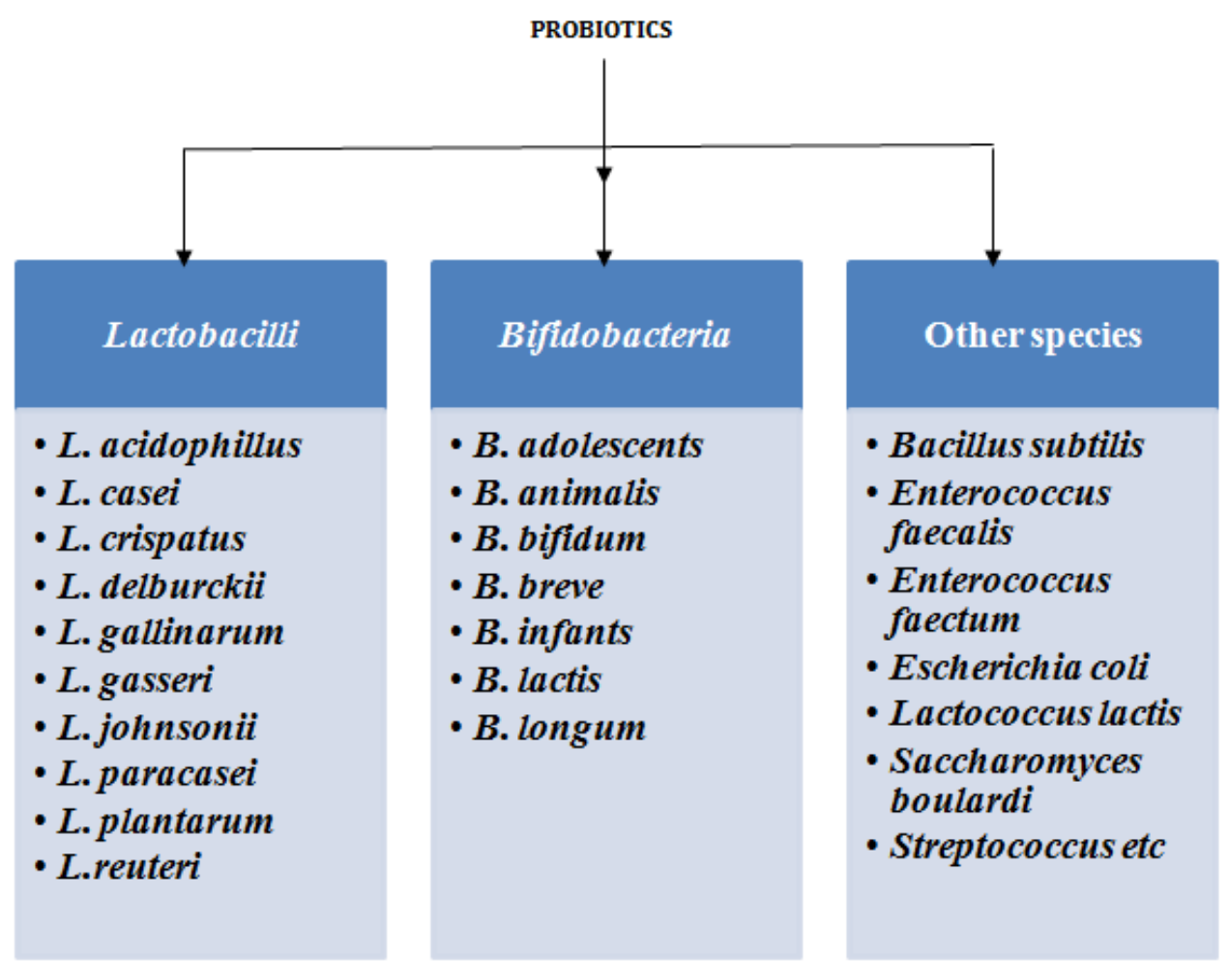

\section{Health Benefits / Efficacy of Probiotics}

Probiotics have a number of health benefits for humans and animals, such as reducing lactose intolerance indication, enhancing the bioavailability of nutrients, endorsement lactose digestion, maintaining gut motility and many more. Probiotics help control intestinal microflora and improve immune function. Probiotics also decrease the pervasiveness of allergies in individuals, inhibit the inflammatory responses in the gut, and have antagonistic effects adjacent to intestinal and food-borne pathogens such as E.coli., Staphylococcus aureus and many more (Holzapfel WH, Haberer P et al. "Overview of gut flora and probiotics”. Int J of Food Microbiol 1998;41:85-101)

1. Increased nutritional assessment (better digestibility, increased absorption of vitamins and minerals);
2. Endorsement of intestinal lactose digestion;

3. Encouraging influence on intestinal and urogenital flora (antibiotics and radiation induced colitis, yeast infections and vaginitis in women);

4. Regulation of gut motility (constipation, irritable bowel syndrome);

5. Decreased frequency and duration of diarrhea (antibiotic associated, Clostridium difficile, travelers, and rotaviral);

6. Maintenance of mucosal veracity;

7. Enhancement of immune system;

8. Anti-carcinogenic, anti-mutagenic and anti-allergic activities;

9. Sensation of well-being; 


\section{Anti-Candida properties;}

11. Assist in the maintaining of inflammatory digestive conditions such as Inflammatory Bowel Disease (IBD), Crohn's disease and Ulcerative Colitis and Interstitial Cystitis

12. Relieving urinary region infections;

13. Optimistic influence on autistic children;

14. Provides antagonistic environment for pathogens for e.g. E.coli;

15. Blocking bond sites from pathogens; and

16. Inactivating enterotoxins.

Bacteria typically colonize the intestinal tract first and then reinforce the host defense systems by inducing generalized mucosal immune responses. Reports indicate that lactic acid bacteria (LAB) as Lactobacillus and Bifidobacterium and their fermented products are effective at enhancing innate and adaptive immunity, prevent gastric mucosal lesion development, alleviate allergies, and put up defense against intestinal pathogen infection.

\section{Efficacy of Probiotics depends upon several factors}

1 Dose administration

2 Mode of administration

3 Strains

4 Duration of treatment

5 Health status of individual

The diagram below is a good representation of various functions and health benefits of probiotics. (Holzapfel $\mathrm{WH}$, Haberer P et al. "Overview of gut flora and probiotics". Int J of Food Microbiol)

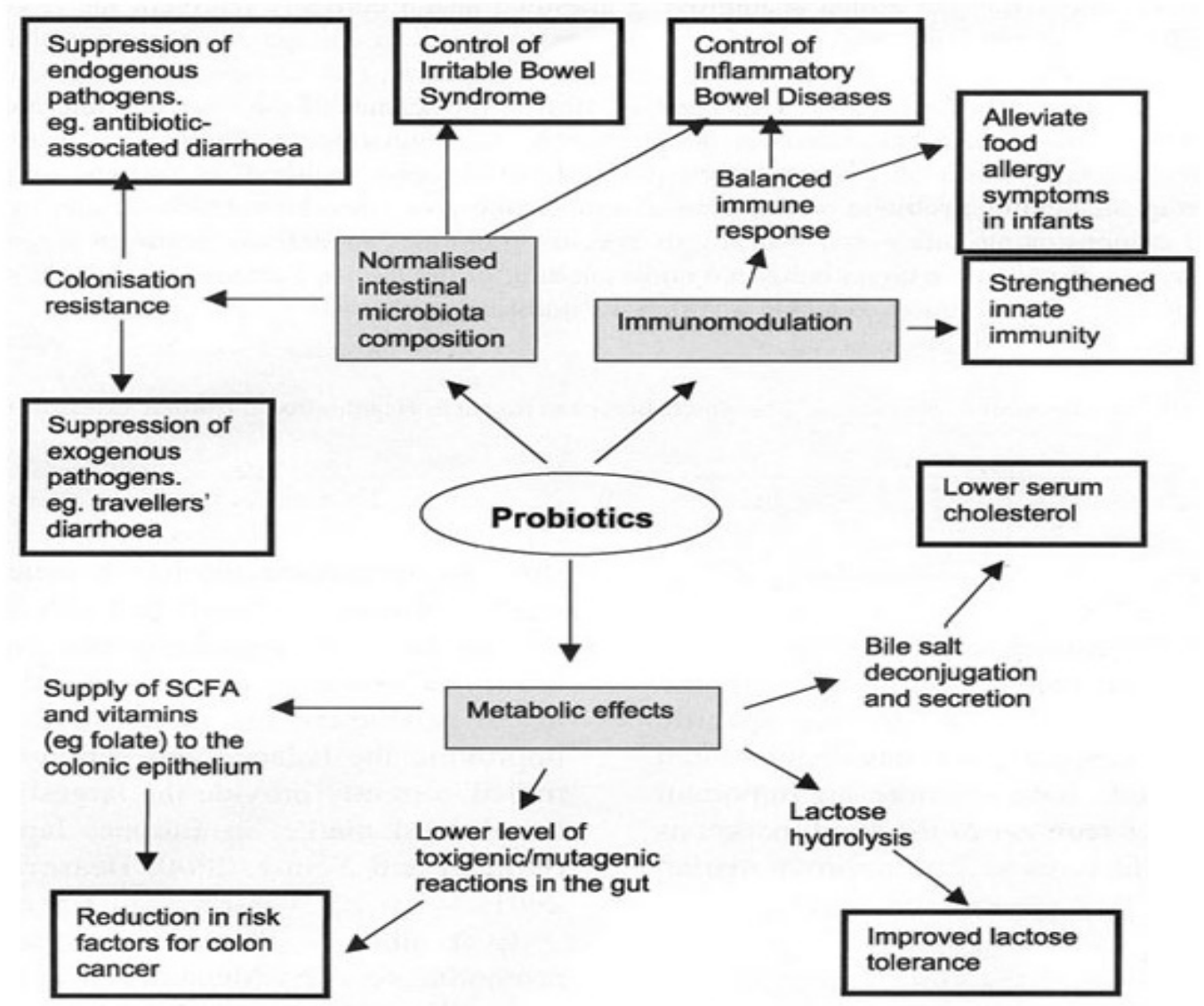

Proposed health benefits stemming from probiotic consumption. 
Probiotics play an vital role in human nutrition and health, and in balancing the intestinal microflora naturally. Health benefits attributed to the consumption of probiotics include: protection of the normal gut flora, improvement of lactose (milk-sugar) intolerance, improvement of digestive processes and assimilation of nutrients, and stimulation of body's immune system. Probiotics are widely used in a lot of countries by consumers and in clinical practice and their health benefits are being examine widely, especially in the last few decades. The need to find alternative therapeutic approaches to overcome side effects associated with the current pharmacological treatments, and the require for new antimicrobials due to the overuse of antibiotics has drive forward research on probiotics against a mass of disorders of varying severity given their favorable safety profiles (Mackie R., Gaskins H.R., "Gastrointestinal microbial ecology". Science \& Medicine Nov./Dec. 1999)

\section{It May Help Reduce Cholesterol}

High cholesterol levels may increase the menace of heart disease. This is especially true for "bad" LDL cholesterol. Fortunately, studies suggest that certain probiotics can assist reduce cholesterol levels and that $\mathrm{L}$. acidophilus may be more valuable than other types of probiotics. Some of these studies have examine probiotics on their own, whereas others have used milk drinks fermented by probiotics. One study found that taking L. acidophilus and another probiotic for six weeks considerably lowered total and LDL cholesterol, but also "good" HDL cholesterol. A similar six-week study establish that $L$. acidophilus on its own had no effect. However, there is evidence that stick together Lactobacillus. acidophilus with prebiotics, or heavy carbohydrates and other sources that help healthy bacteria plough can help boost HDL cholesterol and lower blood sugar. This has been confirmed in studies using probiotics and prebiotics, both as supplements and in fermented milk drinks.

\section{It May Prevent and Reduce Diarrhea}

Diarrhea affect people for a number of reasons, including bacterial infections. It can be dangerous if it last a long time, as it results in watery loss and, in some cases, dehydration. A number of observations have shown that probiotics may assist prevent and reduce diarrhea that's connected with various types of diseases. Evidence on the ability of L. acidophilus to treat acute diarrhea in children. Some studies have shown a beneficial effect. One meta-analysis involving more than 200 children found that probiotics help diminish diarrhea, but not only in hospitalized brood. What's further when harried in combination with another probiotic, L. acidophilus may help reduce diarrhea caused by radiotherapy in mature cancer patients. Similarly, it may help decrease diarrhea associated with antibiotics and a common infection called Clostridium difficile, and Staphylococcus aureus and many more. Diarrhea is also common in people who take a trip to different countries and are exposed to new cuisine and environments. A review of 12 studies found that probiotics are successful at preventing traveler's diarrhea and that Lactobacillus acidophilus, in grouping with another probiotic, was most effectual on doing so.

\section{It Can Improve Symptoms of Irritable Bowel Syndrome}

Irritable bowel syndrome (IBS) affects up to one in five people in many countries. Its sign include abdominal pain, bloating and curious bowel movements. Some research suggests that IBS it might be caused by certain types of bacteria in the intestines. Therefore, a numeral of studies have examined whether probiotics can help recover its symptoms. In a study in 70 people with functional bowel disorders including IBS, taking a combination of probiotic for one to two months improved bloating. A similar study found that Lactobacillus acidophilus alone also reduced abdominal pain in IBS patients. On the other hand, a study that examined a mixture of Lactobacillus acidophilus and other probiotics found that it had no effect IBS symptoms. This might be explained by another study signifying that taking a low dose of single-strain probiotics for a short duration may recover IBS symptoms the most. Specifically, the study indicate that the best way to take probiotics for IBS is to use single-strain probiotics, rather than a mix, for less than eight weeks, as well as a dose of less than 11 billion colony-forming units (CFUs) per day. However, it's important to choose a probiotic supplement that has been scientifically proven to profit IBS.

\section{It Can Help Treat and Prevent Vaginal Infections}

Vaginosis and vulvovaginal candidiasis are the most common types of vaginal infections. There is virtuous evidence that Lactobacillus acidophilus can help treat and prevent such infections. Lactobacilli are classically the most common bacteria in the vagina. They generate lactic acid, which avoid the growth of other harmful bacteria. However, in cases of certain vaginal disorders, other species of bacteria begin to outnumber lactobacillus species. A number of studies have found taking probiotic supplement can prevent and treat vaginal infections by increasing lactobacilli in the vagina. Nevertheless, other observations have found no effect. Eating yogurt that enclose various probiotic bacteria may also prevent vaginal infections. Until now both of the studies that scrutinize this were quite small and would need to be simulated on a larger scale before any assumption could be made.

\section{It May Help Prevent and Reduce Symptoms of Eczema}

Eczema is a situation in which the skin becomes red-looking, resulting in itchiness and pain. The most ordinary form is called atopic dermatitis. Verification suggests that probiotics can lessen the symptoms of this inflammatory condition in both adults and children. Several study set up that giving a mix of certain probiotics bacteria (such as Lactobacillus acidophilus and many others suchas bifidobacteri species ) to pregnant women and their infants during the first three months of life reduced the occurrence of eczema by $25 \%$ by the time the infants reached approximately one year of age . A comparable study found that L. acidophilus, in combination with traditional medical therapy, significantly improved atopic dermatitis symptoms in children. However, not all studies have shown optimistic effects. A large study in 235 newborn children given Lactobacillus acidophilus for the first six months of life found no valuable effect in cases of atopic dermatosis. In fact, it increased sensitivity to allergen 


\section{It's Good for Your Gut Health}

Your gut is lined with trillions of bacteria that play an significant role in your health. Generally, lactobacillus species are very good for gut health. They produce lactic acid, which may prevent injurious bacteria from inhabit the intestines. They also ensure the lining of the intestines stays intact. L. acidophilus can amplify the amounts of other healthy bacteria in the gut, including other lactobacilli and Bifidobacteria. It can also enlarge levels of short-chain fatty acids, such as butyrate and many more, which promote gut health. Another study carefully examined the effects of Lactobacillus acidophilus on the gut. It found that taking it as a probiotic increased the appearance of genes in the intestines that are involved in immune response. These results suggest that probiotics may support a healthy immune system. A separate study examined how the amalgamation of L. acidophilus and a prebiotic affected human gut health. It found that the combined supplement increased the amounts of lactobacilli and Bifidobacteria in the intestines, as well as branched-chain fatty acids, which are an significant part of a healthy gut.

\section{Conclusion}

The microbiome is an assortment of all microbial species that coexist within an individual. These organisms manipulate several aspects of individual body functions. Probiotic organisms are generally beneficial components of microflora and confer normal health statusin the well being. Usually, probiotics should be provided from the exterior in the diet for maintaining suitable health status in today,s world. Probiotics are extensively used worldwide due to advancement in the affiliation between nutrition and health besides their promising benefits and approximately, negligible sideeffects. Indian probiotic industry is achieving its rapidity at steady state with oppurtinities for quick growth in near future. The future of probiotics / probiotic foods is even shows a potential, as current consumers/people are worried to sustain their personnel health and expect that they eat to be healthy and capable of preventing illness. This paper offers a brief overview of the health benefits of probiotics as therapeutic agents.

\section{Reference}

1. Green BK and Schleicher L: US patent, 2800457, CA 1957,51;15842d 1957; 13-627.

2. Ansel HC, Pharmaceutical dosage form and drug delivery system. Lippincott Williams and Wilkins. 2000;233-234.

3. Yazici E, Oner, Kas HS, Hincal AA. Phenytoin sodium microcapsules: bench scale formulation, process characterization and release kinetics. Pharmaceutics Dev Technol. 1996;1:175-183. Doi:10.3109/10837459609029892

4. Blair HS, Guthrie J, Law T, Turkington P Chitosan and modified chitosan membranes I, preparation and characterisation. J App Poly Sci. 1987;33:641-656. Doi: 10.1002/app.1987.070330226

5. Nack H, Microencapsulation techniques, application and problems. J.Soc.Cosmetic Chemists. 1970;21:85-98.

6. Swapan Kumar Ghosh. Functional Coatings and Microencapsulation: A General Perspective. WILEY-VCH Verlag GmbH \& Co. KGaA, Weinheim. 2006. Doi: $10.1002 / 3527608478 . c h 1$

7. Finch CA, Polymers for microcapsule walls. Chem. Ind. 1985;22:752756.

8. Li SP, Kowarski CR, Feld KM and Grim WM Recent advances in microencapsulation technology and equipment. Drug Dev Ind Pharm. 1988;14(2-3):353-376. Doi: 10.3109/03639048809151975

9. Lehman Leon, Lieberman A. Herbert and Kanig L.Josep. The Theory and Practice of Industrial Pharmacy. 3rd edition, Vargehese Publishing House. 1976;412.

10. Schwendeman SP. Recent advances in the stabilization of proteins encapsulated in injectable PLGA delivery systems. Crit Rev Ther Drug Carrier Syst. 2002;19(1):73-98. 